\title{
MODELO DE CONSUMO DE VIVIENDA ÓPTIMO: ESTIMACIONES PARA BOGOTÁ*
}

\author{
MILTON CAMELO RINCÓN" \& JACOBO CAMPO ROBLEDO \\ UNIVERSIDAD CATÓLICA DE COLOMBIA
}

Recibido/ Received/ Recebido: 23/04/2015-Aceptado/ Accepted / Aprovado: 15/06/2015

\begin{abstract}
Resumen
Este artículo presenta una breve revisión del sector de la vivienda en Bogotá, destacando que la política se ha enfocado primordialmente en el impulso a la demanda mediante el otorgamiento de subsidios y acceso al crédito hipotecario. La evolución reciente del mercado sugiere que la política efectuada no garantiza un consumo de vivienda óptimo. En este sentido, se propone un modelo teórico de valores óptimos de demanda, así como la estimación empírica de dichos valores a partir de datos de la Encuesta de Capacidad de Pago realizada en Bogotá en el año 2005. Los resultados muestran brechas importantes entre estratos y sugieren que los niveles de financiación necesarios para una política de demanda pueden resultar excesivamente altos. Se justifica así la pertinencia de una política que no sea enfocada necesariamente a la compra de vivienda, sino que se oriente al uso eficiente de la oferta actual.
\end{abstract}

Palabras clave: Demanda; Bienestar social; Política; Vivienda.

\section{OPTIMAL HOUSING CONSUMER MODEL: ESTIMATES FOR BOGOTÁ}

\begin{abstract}
This article presents a brief review of the housing sector in Bogota, noting that the policy has focused primarily on boosting demand by granting subsidies and access to mortgage credit. The recent market evolution suggests that the policy made no guarantees for an optimal housing consumption. In this sense, a demand of optimal values theoretical model is proposed and the empirical estimation of these values from an Affordability Survey data held in Bogota in 2005. The results show significant gaps between strata and They suggest that funding levels required for a demand policy may be excessively high. The relevance of a policy that is not necessarily focused on the purchase of housing, but is oriented to the efficient use of the current supply is thus justified. Keywords: Demand; Social Welfare; Politicy; Housing.
\end{abstract}

\footnotetext{
Artículo de investigación.

Docente - Investigador, Facultad de Ciencias Económicas y Sociales de la Universidad de la Salle. Correo electrónico: mscamelo@unisalle.edu.co

*... Docente - Investigador, Facultad de Ciencias Económicas y Administrativas de la Universidad Católica de Colombia. Dirección postal: Cra. 13 \# 47 - 49 Piso 3 - Bloque C Facultad de Economía - Universidad Católica de Colombia. Teléfono: (1) 3277300 ext 5155. Correo electrónico: jacampo@ucatolica.edu.co
} 


\title{
MODELO DE CONSUMO ÓTIMO DE MORADIA: ESTIMATIVAS PARA BOGOTÁ
}

\begin{abstract}
Resumo
Este artigo apresenta uma breve revisão do setor da moradia em Bogotá, destacando que a política se focou primordialmente no impulso à demanda mediante a outorga de subsídios e acesso ao crédito hipotecário. A evolução recente do mercado sugere que a política efetuada não garante um consumo ótimo de moradia. Neste sentido, propõe-se um modelo teórico de valores ótimos de demanda, bem como a estimativa empírica de ditos valores a partir de dados da Pesquisa de Opinião de Capacidade de Pagamento realizada em Bogotá no ano de 2005. Os resultados mostram brechas importantes entre estratos e sugerem que os níveis de financiamento necessários para uma política de demanda podem resultar excessivamente altos. Justifica-se assim a pertinência de uma política que não seja focada necessariamente na compra da moradia, sina que se oriente ao uso eficiente da oferta atual.
\end{abstract}

Palavras chave: Demanda; Bem-estar social; Política; Moradia.

Camelo, M. \& Campo, J. (2017). Modelo de consumo de vivienda óptimo: estimaciones para Bogotá. En: Revista de la Facultad de Ciencias Económica: Investigación y Reflexión. rev.fac. cienc.econ, XXV (1). DOI: http://dx.doi.org/10.18359/rfce.2655

JEL: $R 21, R 31, R 52$.

\section{Introducción}

La problemática de vivienda en Bogotá trasciende lo meramente político para inscribirse como una preocupación de la academia y la sociedad en general. De ahí la importancia de generar aportes que permitan enriquecer el conocimiento de la problemática en la ciudad de Bogotá y así construir soluciones efectivas, especialmente dirigidas a subsanar la baja calidad de vivienda de gran parte de la población de la capital. Es tan relevante este tema que según las estadísticas oficiales (DANE) más del 30\% del gasto de los hogares colombianos se destina a consumo asociado con la vivienda, superior que la participación del consumo en alimentos, el cual llega al 28\% del total. Este 30\% contempla gastos como servicios públicos, muebles, productos del aseo del hogar, etc., no obstante, sólo los gastos de arrendamiento (efectivo e imputado) superan el 15\% del gasto total de la economía. Estos últimos, más que la compra de vivienda, es el que asumimos en este documento como consumo de vivienda, pues recoge el gasto en vivienda durante un periodo específico y no la inversión propiamente dicha.

En este sentido, más que alcanzar niveles óptimos de consumo de vivienda, el papel del Estado en Colombia se ha concentrado históricamente en la provisión de vivienda nueva para lograr objetivos de cobertura y reducción del déficit cuantitativo (Vergara, 2008). Por lo tanto, existe una aparente falta de preocupación por los niveles de calidad de vivienda y sus implicaciones sobre el bienestar de la población. Es por esta razón que este documento utiliza un enfoque formal para describir el comportamiento de los hogares en su decisión óptima de gasto en vivienda, para así lograr una aproximación sobre el grado de financiación que sería necesario para una política efectiva concentrada en el consumo y no en la propiedad. Esto se basa en la premisa de que el Estado continúa con una política de demanda en la que actúa más como subsidiario que como promotor de políticas estructurales. Evidentemente, la hipótesis de partida es que una política de estado 
subsidiario es insostenible, de forma que sería necesario considerar alternativas de política más allá de la demanda.

Además de esta introducción, el documento se organiza en las siguientes secciones. En la segunda sección se presentan algunos fundamentos teóricos que ilustran el método formal para evaluar el consumo de vivienda, en la tercera sección se describen algunos antecedentes del mercado de vivienda y el enfoque de demanda de la política en torno al sector. La cuarta sección propone y formaliza un modelo teórico de demanda que permite establecer valores óptimos de consumo por tipo de hogar. En la quinta parte se utiliza el modelo anterior para estimar empíricamente la necesidad de financiamiento por hogar en busca de maximizar el bienestar del mismo. Por último, en la última sección se concluye y resumen los resultados derivados del documento.

\section{Fundamentos teóricos}

En cuanto a los fundamentos teóricos de este trabajo, la teoría económica es rica en modelos formales que intentan explicar el comportamiento de los hogares bajo supuestos de racionalidad y optimización. Es posible considerar como aportes tradicionales en torno a la dinámica de consumo y el papel del sector público en la provisión de bienes meritorios los de Samuelson (1954), Tiebout (1956), Downs (1957) y Black (1958), entre otros. Estos aportes se basan en modelos estáticos de elección pública o social en la que es posible determinar un nivel óptimo de gasto en bienes públicos (en nuestro caso vivienda). En muchos de estos modelos el componente de la vivienda es fundamental en las decisiones de consumo y bienestar de los hogares.

Un planteamiento formal de un modelo con propiedad y consumo de vivienda, puede estudiarse en Rubinfeld (1987) y Henderson (1979); se plantea entre otros argumentos, que una política de vivienda debe ser sostenible financieramente para el gobierno, de forma que necesariamente debe existir un esfuerzo económico por parte de los habitantes de una comunidad. En la misma dirección, Hamilton (1975) había planteado que las comunidades imponen un mínimo consumo de vivienda, hacien- do que los individuos sean homogéneos en términos de ingresos, a la vez que eliminaba el problema del free-rider ${ }^{1}$. La idea central es que la vivienda es un bien meritorio que el estado debe garantizar a la población, sin embargo, esta última debe asumir una carga (generalmente impositiva) que permita que dicha provisión sea factible y eficiente.

A pesar de la consideración sobre la vivienda como un bien meritorio, es claro que la propiedad genera cierto grado de capitalización de los hogares, esta surge cuando el valor de la propiedad aumenta como resultado de la estructura fiscal del gobierno.

Aquí entra en juego la disposición a pagar por la tierra (vivienda) en cada comunidad y la cantidad de servicios públicos que esté proporcionando el estado en relación a la carga impositiva. Cuando se incorpora este tema en nuestro marco de análisis, la relación entre consumo de vivienda y política pública es estrecha, debido a que lo segundo, se supone, es financiado por impuestos al valor de la vivienda (Epple \& Visscher, 1978; Yinger, 1980; Rubinfeld, 1987; Yinger et al., 1988). Ahora bien, no es posible hablar de un consenso teórico sobre una política de vivienda optima, pues los resultados dependen de temas subyacentes como el número y tamaño de las comunidades, el grado de elasticidad en la oferta de la tierra, la distorsión generada por los impuestos a la propiedad, los inventivos de los hogares a capitalizar, el grado de movilidad de los hogares, etc. (Camelo, 2009). Sin embargo, sí se han realizado estudios empíricos que dan luces sobre las implicaciones de la política gubernamental en las decisiones de consumo de vivienda de los hogares.

En concordancia con lo anterior, la función de utilidad del tipo Stone (1954) ha sido utilizada en numerosas ocasiones para estimar empíricamente los efectos de los programas de gobierno sobre el consumo de los hogares. Esta herramienta teórica ha sido popular en la medida que cumple tres criterios fundamentales para ser usada: i) proporcionar una aproximación aceptable al proceso de estudio;

Este concepto está asociado a los incentivos que tienen los individuos de consumir bienes y servicios públicos sin asumir una carga impositiva proporcional. 
ii) poder ser aplicado con datos existentes; $y$ iii) debe ser computable (Stone, 2014).

Trabajos como los de Awan, Odling-Smee \& Whitehead (1982) y De Borger (1984, 1986), especifican una función de utilidad Stone Geary definida por atributos de vivienda y otros bienes, con el fin de estimar ecuaciones de demanda a partir de parámetros estructurales. Se pretendía evaluar los beneficios y efectos sobre el consumo de programas de vivienda pública, encontrando en diferentes contextos, que los programas (generalmente basados en subsidios) tienen efectos sobre el consumo de vivienda y de otros bienes, explicado por los efectos renta y sustitución. Más recientemente, este tipo de análisis lo realizó Schmidheiny (2006), pero esta vez calibrando un modelo donde los hogares difieren en ingreso y preferencias sobre la vivienda. El resultado básico de este último, es que existe una segregación imperfecta que depende del grado de sustituibilidad entre bienes públicos y privados.

Finalmente, cabe destacar que aunque la metodología que se propondrá aquí no ha sido de aplicación exclusiva en el caso de demanda de vivienda, lo cual puede evidenciarse en trabajos recientes que analizan temas distributivos y de consumo de bienes meritorios como el agua (Schmidheiny, 2006; Renzetti, et al., 2015; Achury, et al., 2012), una de las principales aplicaciones ha sido, en efecto, el consumo de la vivienda. Este es un bien muy particular, que si bien comparte características con otros bienes, no las comparte todas: es un bien meritorio, durable, heterogéneo, inmóvil espacialmente, entre otras (Smith, et al., 1988). Por lo tanto, el estudio del consumo de vivienda puede realizarse desde diferentes enfoques y consideraciones ${ }^{2}$. En este sentido, la hipótesis del ciclo de vida de Modigliani, puede dar nociones acerca de las preferencias de los hogares en este bien. Biorn (1980) realizó un interesante trabajo aplicado donde contrasta la hipótesis de ciclo de vida de Modigliani haciendo uso de la función de utilidad Stone Geary,

2 Por ejemplo, un enfoque interesante que está fuera del alcance de este trabajo es el efecto riqueza de la vivienda y su importancia en el ciclo vital del consumo de los hogares. Este argumento se relaciona con los argumentos de Ando \& Modigliani (1963) y Hall (1978), y ha sido desarrollado en trabajos como los de Biorn (1980), Cocco (2005) y Lopez \& Salamanca (2009). para analizar la relación entre gasto de consumo, ingreso y salud a nivel microeconómico por edades. Este es un tema que sigue siendo pertinente, pero cuyas respuestas dependerán de los estudios que se hagan en cada región en particular.

\section{El enfoque de demanda de la política de vivienda}

El estimuló al sector de la construcción ha sido uno de los puntos clave de la política de vivienda en Bogotá, siendo este sector uno de los principales indicadores de la actividad económica en el corto plazo. Sin embargo, esto no significa necesariamente que la política de vivienda genere grandes impactos en el resto de la economía, pues sin incluir obras civiles, la construcción representaba en 2014 tan sólo el 5,3\% del PIB; probablemente, de lo que se trata, es de un sector altamente vinculado a los vaivenes de la demanda agregada. El reconocimiento de este hecho toma validez al observar la estrecha relación entre el comportamiento del mercado de vivienda y el del PIB total de la economía (Ilustración 1).

El mercado de la vivienda tiene la particularidad de presentar una oferta limitada, siendo el suelo su principal componente, de forma que se puede hablar de una oferta inelástica. Solamente cambios estructurales en la disponibilidad del suelo o legislación territorial podrían afectar las condiciones de mercado desde la oferta, sin embargo, esto no parece ser lo ocurrido en los últimos años. En ese orden de ideas, la evolución de los precios es atribuible principalmente a las condiciones de demanda o a posibles comportamientos especulativos en el sector. La Ilustración 2 ilustra la evolución del índice de precios de apartamentos nuevos, el índice de precios de casas nuevas, el índice de precios de vivienda usada (casas y apartamentos) y el índice de costos de construcción de vivienda ${ }^{3}$; cada uno de ellos calculado en términos reales (aislando la infla-

\footnotetext{
El índice de precios de vivienda nueva (casas y apartamentos) es elaborado por el Banco de la República y consiste en un promedio simple del precio por metro cuadrado de todos los inmuebles nuevos, de ahí que no hace consideraciones de calidad. El Índice de precios de la vivienda usada y el índice de costos de construcción son calculados por el DANE. Los índices reales se calcularon corrigiendo por IPC total.
} 
ción del resto de la economía). A pesar de la relativa estabilidad de los costos de construcción durante la última década y media, los precios han crecido significativamente desde el año 2004. Esta evolución sugiere un posible exceso de demanda de vivienda durante dicho periodo.

El mayor crecimiento de los precios se ha notado en los apartamentos nuevos y casas usadas, duplicándose desde 2003 hasta 2014, mientras los precios de las casas nuevas tuvieron un crecimiento levemente inferior. Por su parte los costos de construcción en términos reales llegaron, incluso, a descender levemente. Estos hechos estilizados, así como diferentes trabajos empíricos en torno al problema, sugieren la existencia de una burbuja de demanda en el sector (Sánchez \& Vega, 2015; Gómez, et al., 2015; Camelo \& Campo, 2016).

En cuanto a la política de vivienda ${ }^{4}$, concretamente, los antecedentes se remontan a la creación de Banco Central Hipotecario (BCH) en 1932, con el cual el gobierno nacional pretendía canalizar recursos hacia la financiación de vivienda popular. En 1939 fue creado el Instituto de Crédito Territorial (ICT) cuya función era ejecutar proyectos de construcción y financiación de vivienda, principalmente de interés social (VIS). Dichas entidades funcionaron con algunas transformaciones durante el resto del siglo $\mathrm{XX}$ fomentando el acceso de vivienda de la clase trabajadora ${ }^{5}$. A partir de 1972 entró en funcionamiento el conocido sistema UPAC, el cual buscaba que el dinero captado y canalizado hacia la compra de vivienda fuera calculado con base en una unidad de poder adquisitivo constante, es decir, que se ajustara de acuerdo al comportamiento de la inflación (Echeverri, García \& Pineda, 1999).

El sistema generó resultados positivos al dinamizar el sector de la construcción, en un entorno en el

$4 \quad$ El breve análisis de esta sección constituye una síntesis de parte de un trabajo de los autores donde se demuestra con mayor detalle los argumentos. Para profundizar en los mismos ver Camelo \& Campo (2016).

5 El BCH fue liquidado en el año 2000, mientras que el ICT se convirtió en el Instituto Nacional de vivienda de interés social y reforma urbana (INURBE) en 1991. que aún era fácil encontrar suelo urbanizable a bajo costo en la ciudad de Bogotá. Se sumaba a esta iniciativa la importancia de la Caja de Vivienda Popular (CVP), la cual promovió grandes proyectos de construcción particularmente para poblaciones de estratos 1 y 2 en la ciudad. Asimismo son varias las iniciativas hacia el fomento de adquisición de vivienda popular, tal es el caso de políticas como el plan de vivienda sin cuota inicial en el gobierno 19821986 y la reforma urbana de 1989; esfuerzos que permitieron que una parte importante de población de bajos recursos lograran acceder a vivienda propia (Jaramillo, 1992).

Acompañado del paquete de reformas estructurales de tipo neoliberal que vivió la economía en los inicios de la década de 1990, el estado pasa de ejercer un papel de intermediario financiero en el mercado de vivienda a ejercer un papel de subsidiario. Dicho papel lo ejercería mediante el Instituto Nacional de Vivienda de Interés Social y Reforma Urbana (INURBE) y las cajas de compensación que desde entonces operan y asignan los subsidios de vivienda a la población afiliada.

El año de 1997 fue crítico para la economía mundial tras la crisis asiática que restringió el flujo de capitales hacia la región. En Colombia esta crisis se manifestó en fuertes crecimientos en las tasas de interés y la contracción del crédito. El saldo de la deuda hipotecaria de las familias creció a tal punto de superar en algunos casos el precio de los inmuebles, lo cuales venían en descenso. Cuando las tasas de interés comenzaron a crecer más rápido que el IPC, el sistema UPAC se vuelve insostenible y estalla una crisis hipotecaria en el país que afectó la compra de vivienda (Urrutia \& Namen, 2012).

Después de la crisis internacional de 2008 - 2009, el gobierno adoptó una medida de subsidios a la tasa de interés, lo cual constituyó una política contracíclica que reactivó la demanda y el sector desde el año 2010. Este es un ejemplo claro del enfoque de demanda de la política gubernamental en torno al mercado de la vivienda (Camelo \& Campo, 2016). 
Ilustración 1. Crecimiento del PIB total y el sector Construcción en Colombia, 2001 - 2014p

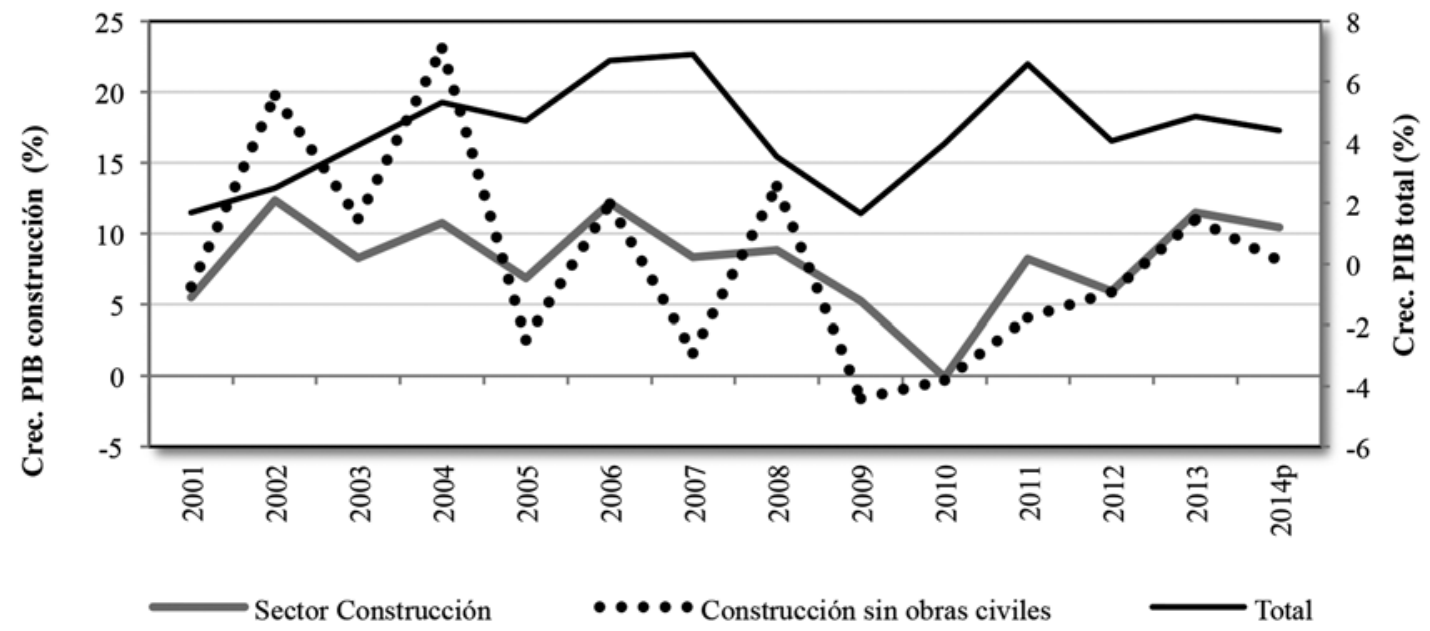

Fuente: DANE, Cuentas Nacionales.

Ilustración 2. Índice real de precios de la vivienda (1997 - 2014)

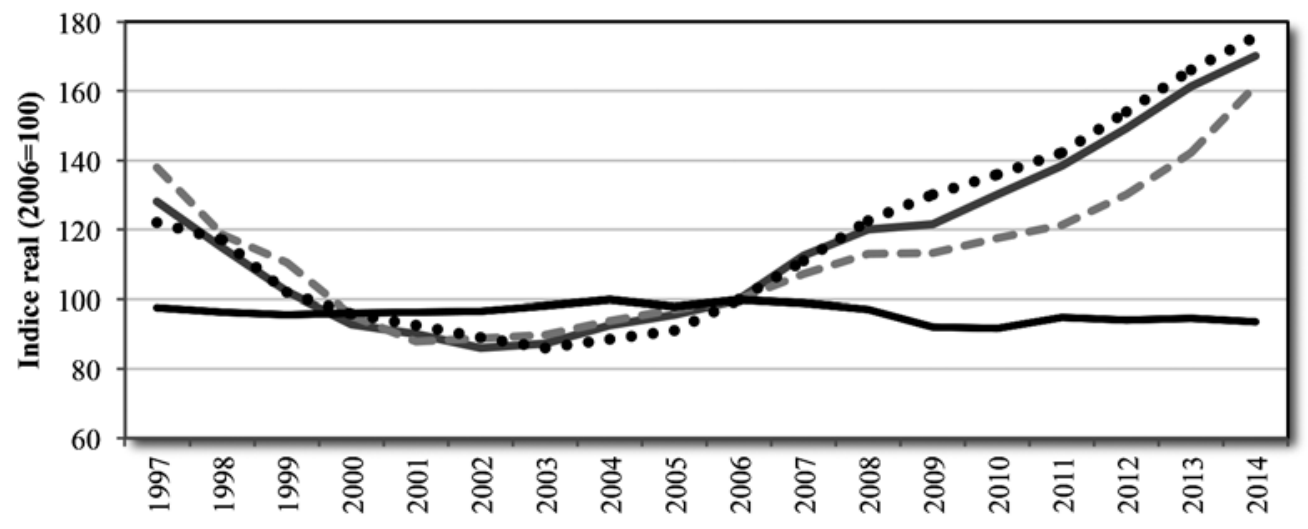

Apartamentos nuevos - Casas nuevas

$\bullet \bullet \bullet \bullet$ Vivienda usada

Indice de costos

* Cálculos propios

Fuente: DANE y Banco de la República.

\section{Un modelo de demanda óptima}

La evidencia sobre una política de vivienda en Colombia enfocada principalmente en la demanda, crea la necesidad de repensar este enfoque a partir de criterios de maximización de bienestar de la población. Como se ha anotado anteriormente, lo política ha sido en cierto grado efectiva en financiar la compra de vivienda, sin embargo, pocas veces se reflexiona sobre las verdaderas preferencias de la 
población en lo que respecta al consumo de vivien$\mathrm{da}^{6}$. Existen alternativas que no necesariamente se limitan a la compra de vivienda, más aún cuando esta se trata de interés social; mecanismos alternativos como el arrendamiento son opciones posibles cuando el objetivo es maximizar un bienestar social. El modelo que se presenta en esta sección asume que los hogares buscan financiar su consumo de vivienda para maximizar su bienestar, más allá de cuestionamientos sobre si es vivienda propia o no.

\subsection{Función de bienestar social}

Se propone un modelo estático de determinación de valores óptimos de gasto en vivienda e impuestos. Existe un planeador central cuyo objetivo es maximizar el bienestar de un individuo representativo de la comunidad, el cual está determinado por dos tipos de bienes: consumo de vivienda (CV) y consumo de otros bienes (OB). El papel del gobierno es determinar los niveles de gasto en cada tipo de bienes y la estructura tributaria necesaria para financiar los mismos.

La formalización de este argumento requiere definir un conjunto mínimo de bienes básicos en donde es indispensable la provisión pública; en otras palabras existe un requisito de consumo de vivienda mínimo. Para incorporar este hecho, se ha optado por utilizar como indicador del bienestar social una función Stone-Geary (1954) de la siguiente forma:

$$
W=\left(C V-\sum \theta_{i}^{C V}\right)^{\alpha}\left(O B-\sum \theta_{j}^{O B}\right)^{\beta}
$$

Donde las variables se definen de la siguiente manera:

W: Bienestar de un individuo representativo de la población.

\footnotetext{
Recientemente el gobierno nacional ha promovido un nuevo plan de vivienda popular consistente en la entrega gratuita de 100.000 viviendas a población de bajos recursos, plan inspirado en modelos de países como Brasil y Chile. Sin embargo, no se trata de una política con un enfoque substancialmente diferente en el sentido que se fundamenta en promover mayor consumo de vivienda financiada por el estado. La disponibilidad de tierra y la adecuada planificación urbanística de las ciudades (oferta de suelo) van mucho más allá de este tipo de políticas (Camelo \& Campo, 2016).
}

$\mathrm{CV}$ : Consumo de Vivienda del individuo representativo.

$\mathrm{OB}$ : Consumo de otros bienes del individuo representativo.

$\theta_{i}^{C V}$ : Cantidad mínima de subsistencia de consumo de vivienda en cada característica i (servicios públicos y condiciones arquitectónicas mínimas).

$\theta_{j}^{O B}$ : Cantidad mínima de subsistencia de consumo de otros bienes tipo $\mathrm{j}$ (alimentos, transporte, salud y educación).

$\alpha$ : Participación del consumo de vivienda sobre la renta que excede el nivel de consumo mínimo.

$\beta$ : Participación del consumo de otros bienes sobre la renta que excede el nivel de consumo mínimo.

Esta definición funcional posee varias ventajas analíticas: i) la función es continua y doblemente diferenciable; ii) es una función con propiedades Cobb - Douglas que, dado $\alpha+\beta=1$, se garantiza la propiedad de utilidades marginales decrecientes, en consecuencia una solución interior (Stone, 1954) y iii) los parámetros son factibles de estimar empíricamente mediante métodos econométricos tradicionales.

De otro lado, dada la dificultad de estimar de manera precisa el consumo en otros bienes $(\mathrm{OB})$ es posible utilizar como variable "proxy" de este gasto el ingreso disponible de los hogares ((1-t)Y). Aunque conceptualmente, este ingreso disponible puede incluir gasto de vivienda, se asume como aproximación de OB bajo el supuesto de que en el pago de impuestos (tY) se encuentra parte del consumo de vivienda del individuo. Este supuesto permite reducir el número de variables del modelo sin distorsionar el resultado dado el carácter ordinal de la función de bienestar. Por lo tanto, la función de bienestar social se puede expresar como sigue:

$$
W=\left(C V-\sum \theta_{i}^{C V}\right)^{\alpha}\left((1-t) Y-\sum \theta_{j}^{O B}\right)^{\beta}
$$

Donde (1-t)Y indica el ingreso disponible del individuo representativo, es decir, renta corriente menos obligaciones tributarias ${ }^{7}$.

\footnotetext{
La renta disponible puede ser superior a la renta total en el escenario de una comunidad local benefactora de transferencias gubernamentales. La política de vivienda óptima puede implicar escenarios como este en comunidades de bajos recursos.
} 


\subsection{La restricción presupuestal}

Los recursos totales disponibles para maximizar el bienestar social están dados por el ingreso disponible de la comunidad, los ingresos tributarios locales, las transferencias del gobierno nacional, y otros ingresos extraordinarios (por ejemplo deuda o donaciones). Estos recursos deben ser asignados eficientemente para el consumo de los dos tipos de bienes establecidos.

De esta manera expresamos la restricción presupuestal como sigue:

$$
C V+(1-t) Y=Y+T r+O I
$$

Donde el lado izquierdo de la ecuación (2) representa el gasto total de un individuo representativo, el cual se descompone en consumo de vivienda (CV) $y$ gasto en otros bienes $((1-t) Y)^{8}$. El lado derecho representa la fuente de ingresos de un individuo representativo, los cuales se componen por los ingresos totales Y (producto de su trabajo o renta corrientes), las transferencias o subsidios recibidos por el gobierno (Tr) y otros ingresos (OI) que pueden ser resultado de deuda adquirida por el individuo representativo o por ingresos extraordinarios como donaciones.

\subsection{Optimización}

Teóricamente hablando, el problema es hallar los valores óptimos de gasto en vivienda y el recaudo necesario tal que se logre el máximo bienestar del individuo. Formalmente el problema es maximizar (1) sujeto a (2), cuyo lagrangiano está expresado como sigue:

$$
L=\left(C V-\sum \theta_{i}^{C V}\right)^{\alpha}\left((1-t) Y-\sum \theta_{j}^{O B}\right)^{\beta}-\lambda\left(C V+(1-t)^{\prime} Y-Y-T r-O I\right)
$$

\footnotetext{
Nótese que entre sus gastos se encuentra implícitamente las obligaciones tributarias del individuo, pues de su ingreso total se descuenta el pago en impuestos (1-t)Y.
}

Resolviendo el problema se obtienen las condiciones de primer orden respecto a cada variable control (aplicando Lagrangiano9:

$$
\begin{gathered}
\frac{\partial L}{\partial C V}=\alpha\left(C V-\sum \theta_{i}^{C V}\right)^{\alpha-1}\left((1-t) Y-\sum \theta_{j}^{O B}\right)^{\beta}-\lambda=0 \\
\frac{\partial L}{\partial t}=\beta\left(C V-\sum \theta_{i}^{C V}\right)^{\alpha}\left((1-t) Y-\sum \theta_{j}^{O B}\right)^{\beta-1}(-Y)+\lambda Y=0 \\
\frac{\partial L}{\partial \lambda}=C V+(1-t) Y-Y-T r-O I=0
\end{gathered}
$$

De las condiciones (3) y (4) obtenemos:

$$
\frac{\alpha\left(C V-\sum \theta_{i}^{C V}\right)^{\alpha-1}\left((1-t) Y-\sum \theta_{j}^{O B}\right)^{\beta}}{\beta\left(C V-\sum \theta_{i}^{C V}\right)^{\alpha}\left((1-t) Y-\sum \theta_{j}^{O B}\right)^{\beta-1}}=1
$$

Simplificando:

$$
\frac{\alpha\left((1-t) Y-\sum \theta_{j}^{O B}\right)}{\beta\left(C V-\sum \theta_{i}^{C V}\right)}=1
$$

Teniendo en cuenta que $\alpha+\beta=1$, si de la ecuación (5) se despeja, por ejemplo (1-t) $Y$, y se reemplaza en (6), reordenando términos se obtiene que:

$$
C V=\alpha(Y+T r+O I)-\alpha \sum \theta_{j}^{O B}+\beta \sum \theta_{i}^{C V}
$$

Utilizando el resultado en (7), reemplazando de nuevo en (5) y reordenando obtenemos:

$$
T=\alpha(Y+T r+O I)-\alpha \sum \theta_{j}^{O B}+\beta \sum \theta_{i}^{C V}-(T r+O I)
$$
Las condiciones de segundo orden que garantizan que el
punto crítico corresponde a un máximo están expresadas en el Hessiano orlado:

$$
H=\left[\begin{array}{lll}
0 & g_{1} & g_{2} \\
g_{1} & L_{11} & L_{12} \\
g_{2} & L_{21} & L_{22}
\end{array}\right]
$$

Donde

$$
\begin{aligned}
g_{1} & =1, g_{2}=-Y \\
L_{11} & =\alpha(\alpha-1)\left(C V-\sum \theta_{i}^{C V}\right)^{\alpha-2}\left((1-t) Y-\sum \theta_{j}^{o B}\right)^{\beta}<0 \\
L_{12} & =L_{21}=\alpha \beta\left(C V-\sum \theta_{i}^{C V}\right)^{\alpha-1}\left((1-t) Y-\sum \theta_{j}^{O B}\right)^{\beta-1}(-Y)<0 \\
L_{22} & =\beta(\beta-1)\left(C V-\sum \theta_{i}^{C V}\right)^{\alpha}\left((1-t) Y-\sum \theta_{j}^{O B}\right)^{\beta-2} Y^{2}<0
\end{aligned}
$$

Se puede observar fácilmente, dado el signo de cada elemento del Hessiano orlado, que $|\mathrm{H}|>0$, por lo tanto, se cumplen las condiciones de un máximo. 
Donde $T$ denota la carga impositiva $(t Y)$ para un individuo representativo. Por lo tanto, (7) y (8) constituyen las soluciones de política óptima

La ecuación (7) implica que un incremento en el ingreso del individuo representativo se asocia con un incremento en el consumo de vivienda óptimo del mismo (siempre que $\alpha>0$ ), de otro lado, mayores niveles de consumo de subsistencia en otros bienes implica menores posibilidades de proveer consumo de vivienda; esta asociación presentaría el mismo coeficiente que el de los ingresos. Finalmente, como es de esperar, mayores niveles de consumo básico de vivienda se acompañan de un consumo de vivienda mayor. La ecuación (8) se interpreta de forma semejante, y con excepción del término - $(\operatorname{Tr}+\mathrm{OI})$, resulta igual a la ecuación (7), la razón radica en que, dada la restricción presupuestal (2), la política debe ser auto sostenible, lo cual implica que un mayor consumo de vivienda está sujeto a una mayor carga impositiva para los habitantes. El termino - $(\mathrm{Tr}+\mathrm{OI})$ señala que esta carga impositiva puede ser aliviada mediante transferencias del gobierno central u otros ingresos o subsidios a los cuales los individuos de la comunidad puedan acceder.

\subsection{Transferencias condicionadas vs. Recursos no condicionados}

El modelo asume que los recursos $(\mathrm{Tr}+\mathrm{OI})$ necesarios para lograr niveles óptimos de vivienda consisten en fuentes de ingresos y transferencias no condicionadas. Una transferencia es no condicionada si su disponibilidad para el receptor es libre, es decir, no se encuentra sujeta al consumo de un bien específico. Esto implica un aumento en la disponibilidad de recursos que pueden ser destinados al consumo de cualquier tipo de bien, lo cual no ocurre cuando se trata de transferencias condicionadas.

En el marco de las políticas públicas de vivienda, es claro que los subsidios y medidas de financiamiento de vivienda actuales son de tipo condicionado, es decir, se genera una disponibilidad de recursos que deben ser destinados exclusivamente al consumo de vivienda (por ejemplo créditos hipotecarios, subsidios a la tasa de interés, etc.). Con base en el modelo propuesto, la Ilustración 3 ilustra el efecto de una transferencia condicionada y una no condicionada sobre el consumo de vivienda y de otros bienes. La restricción presupuestal del hogar representativo está representada por la línea A-B, mientras que la curva $U_{1}$ denota la curva de indiferencia más alta posible dada dicha restricción; por lo tanto, el punto X representa el consumo óptimo de cada hogar sin ningún tipo de subsidios. De otro lado, la restricción presupuestal A-C es aquella que enfrentaría un hogar representativo después de una transferencia condicionada (política actual), donde las posibilidades de consumo se incrementan sesgadamente hacia la vivienda; bajo una elección racional del hogar, Y es la nueva cesta de consumo del hogar generando una utilidad de U2. Finalmente, tenemos la restricción D-E, la cual indica el presupuesto necesario, a precios iniciales (es decir sin distorsiones), para obtener la misma cesta de consumo (Y) que se obtendría bajo una política de subsidios condicionados.

Como se observa en la Ilustración 3, aunque los subsidios condicionados habituales generan un efecto ingreso que podría incrementar la demanda de otros bienes, sus efectos distorsionan las decisiones óptimas de los agentes, modificando los precios relativos de mercado y generando ineficiencias en la asignación. Al pasar de la cesta $\mathrm{X}$ a la cesta $\mathrm{Y}$ los hogares mejoran su bienestar de $\mathrm{U}_{1}$ a $\mathrm{U}_{2}$ y logran consumir un nivel mínimo de vivienda que se considera de subsistencia $\left(\theta_{i}^{\mathrm{CV}}\right)$, sin embargo, existe un efecto sustitución que conlleva a que este resultado no sea óptimo. Nótese que bajo los precios iniciales, el presupuesto utilizado para conseguir la cesta $Y$ es equivalente al que permite obtener la cesta $Z$, la cual genera mayor bienestar al hogar; esto significa que las distorsiones generadas sobre los precios tienden a sesgar las decisiones de consumo sobrevalorando la importancia del consumo de vivienda.

Una política de vivienda basada en financiación y subsidios condicionados, requiere garantizar un nivel de consumo de vivienda de subsistencia $\left(\theta_{i}{ }^{C V}\right)$, sin embargo, el costo de dicha política es la distorsión sobre los precios relativos del mercado que hace que los hogares limiten su consumo en otros bienes para beneficiarse de dicha política. El consumo de otros bienes es menor en la cesta $Y$ que en 
Ilustración 3. Efecto de los subsidios condicionados y no condicionados

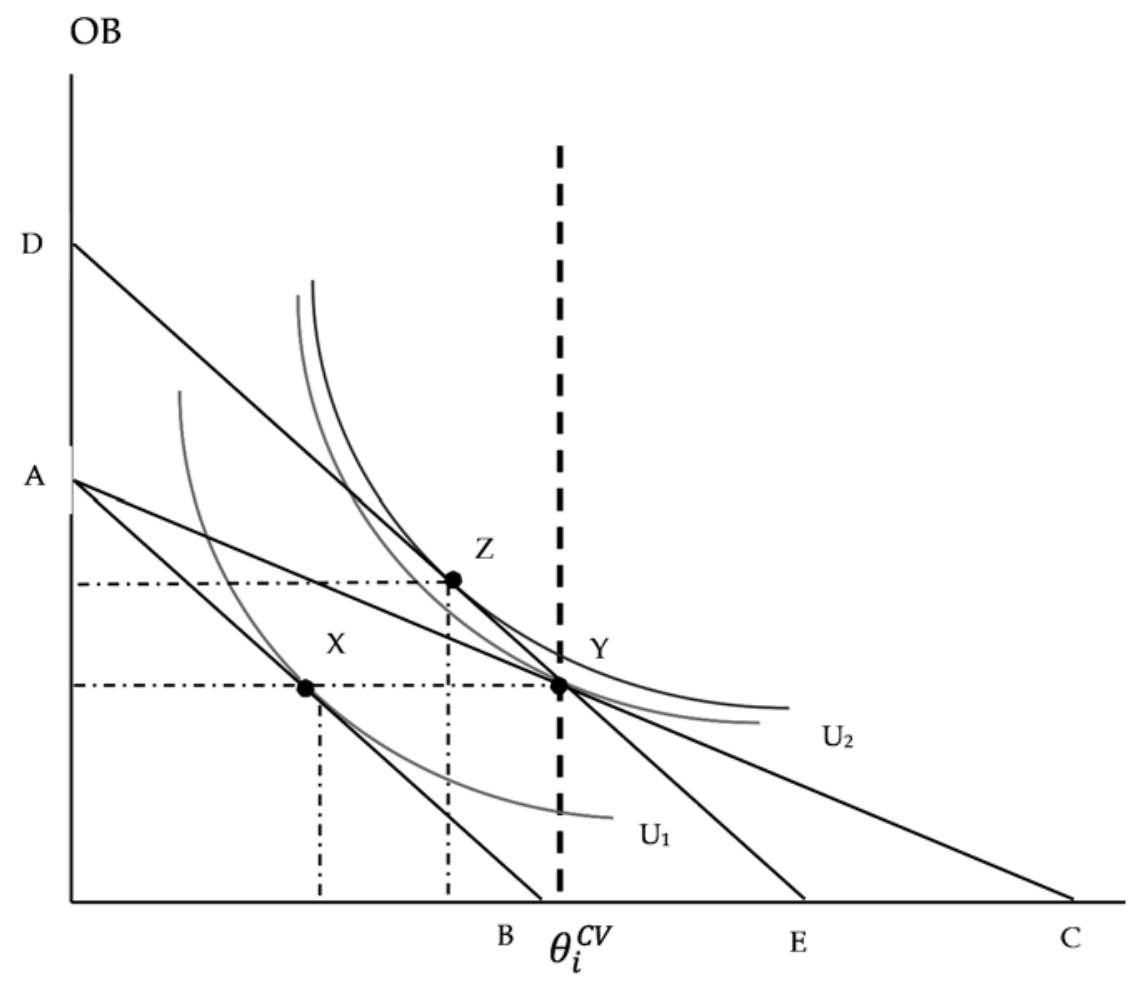

Fuente: Elaboración propia.

la cesta Z, lo cual sugiere que bienes básicos como la educación, la salud, alimentación, etc., pueden ser sustituidos en la práctica por el enfoque de demanda de la política de vivienda. Por su parte, una política de subsidios no condicionada no generaría distorsiones en los precios, por lo tanto se controla automáticamente el exceso de demanda y el exceso de oferta, sin embargo, será más difícil alcanzar los niveles de consumo de vivienda mínimos de subsistencia; esto se clarifica al comparar la cesta $Y$ con la $Z$, pues en la última los hogares no alcanzas a consumir su nivel de vivienda de subsistencia. De la magnitud de la cuantía de los recursos necesarios para garantizar dichos niveles de subsistencia, depende la factibilidad de una política como esta, es así que en la siguiente sección se ilustran algunos resultados empíricos para la ciudad de Bogotá que dan luces al respecto.

\section{Estimaciones empíricas a partir de la ECP 2005}

\subsection{Fuente de datos y variables}

Los datos provienen de la Encuesta sobre Capacidad de Pago de bienes y servicios públicos (ECP) en el Distrito Capital de $2005^{10}$, la cual contiene información de calidad de vida de 12.139 hogares representativos de la cuidad de Bogotá. De los resultados de esta encuesta se tomaron principalmente dos variables: Ingreso mensual por hogar $(\mathrm{Y})$ y carga impositiva por hogar (T). Estas resultan, respecti-

10 La encuesta fue realizada por el Departamento Administrativo de Planeación Distrital (DAPD) y el Centro de Investigaciones para el Desarrollo (CID) de la Universidad nacional de Colombia. 
vamente, de lo que los hogares manifestaron como ingreso mensual y como gasto atribuible a impuestos exclusivamente.

Para efectos e ilustración, la tabla 1 presenta el ingreso por hogar, según localidad, el ingreso per cápita, la carga impositiva y la participación de dicha carga sobre el ingreso. Se observa que la localidad de Usme presentaba el ingreso promedio por hogar más bajo, mientras que Chapinero presentaba el más alto; en cuanto a la carga impositiva, como participación del ingreso, Cuidad Bolívar presenta la baja y Usaquén la más alta.

Para las dos variables utilizadas en las estimaciones, a saber, ingreso por hogar ( $\mathrm{Y}$ ) y carga impositiva ( $\mathrm{T}$ ), se presentan las estadísticas descriptivas básicas en la tabla 2. Se evidencian diferencias significativas en los hogares entre estratos y al interior de estos. Las desviaciones estándar dan luces de la heterogeneidad de los estratos, especialmente en lo referente a la carga impositiva que los hogares manifestaron en la encuesta.

\subsection{Supuestos}

Se parte de los siguientes supuestos sobre las variables involucradas:

- Los valores $\sum \theta_{\mathrm{i}}^{\mathrm{CV}}$ y $\sum \theta_{i}^{\mathrm{OB}}$ son valores exógenos establecidos como los niveles mínimos de "consumo de vivienda" y de "otros bienes" o de subsistencia.

Tabla 1. Ingreso mensual y carga impositiva por localidades (Pesos de 2004)

\begin{tabular}{|c|c|c|c|c|}
\hline Localidad & Ingreso por hogar (Y) & Ingreso per cápita & Carga impositiva (T) & $\%$ Carga impositiva \\
\hline Usme & 630.040 & 202.345 & 34.585 & $5,5 \%$ \\
\hline Ciudad Bolívar & 746.559 & 236.708 & 27.078 & $3,6 \%$ \\
\hline Bosa & 814.576 & 264.331 & 51.628 & $6,3 \%$ \\
\hline San Cristóbal & 881.334 & 338.782 & 50.440 & $5,7 \%$ \\
\hline Rafael Uribe & 881.984 & 311.376 & 62.188 & $7,1 \%$ \\
\hline Tunjuelito & 925.217 & 351.804 & 97.597 & $10,5 \%$ \\
\hline Santa Fé & 1.091 .804 & 578.506 & 75.030 & $6,9 \%$ \\
\hline Kennedy & 1.193 .218 & 408.049 & 99.601 & $8,3 \%$ \\
\hline Mártires & 1.295 .235 & 524.750 & 181.096 & $14,0 \%$ \\
\hline Antonio Nariño & 1.311 .147 & 504.091 & 258.238 & $19,7 \%$ \\
\hline Puente Aranda & 1.439 .653 & 516.192 & 184.487 & $12,8 \%$ \\
\hline Engativa & 1.472 .295 & 542.971 & 169.814 & $11,5 \%$ \\
\hline Candelaria & 1.697 .885 & 888.671 & 132.774 & $7,8 \%$ \\
\hline Barrios Unidos & 1.846 .897 & 839.743 & 325.547 & $17,6 \%$ \\
\hline Fontibón & 2.252 .088 & 884.648 & 201.497 & $8,9 \%$ \\
\hline Suba & 2.416 .329 & 994.547 & 335.400 & $13,9 \%$ \\
\hline Teusaquillo & 2.671 .739 & 1.149 .524 & 419.484 & $15,7 \%$ \\
\hline Usaquén & 3.593 .498 & 1.490 .769 & 730.967 & $20,3 \%$ \\
\hline Chapinero & 4.090 .844 & 2.429 .371 & 536.452 & $13,1 \%$ \\
\hline
\end{tabular}

Fuente: Cálculos propios a partir de la ECP, 2005. 
Tabla 2. Resumen de estadísticas de Ingreso e impuestos (Pesos de 2004)

\begin{tabular}{|l|c|c|c|c|c|c|}
\hline \multicolumn{1}{|c|}{ Estrato } & $\mathbf{1}$ & $\mathbf{2}$ & $\mathbf{3}$ & $\mathbf{4}$ & $\mathbf{5}$ & $\mathbf{6}$ \\
\hline Número de hogares $(\mathrm{n})$ & 2.287 & 4.982 & 3.565 & 757 & 352 & 196 \\
\hline Promedio Ingreso por hogar $(\mathrm{Y})$ & 704.076 & 931.157 & 1.642 .232 & 3.529 .347 & 5.089 .297 & 7.708 .304 \\
\hline Desviación estándar de Y & 508.283 & 742.260 & 1.441 .776 & 3.195 .341 & 3.631 .024 & 6.797 .934 \\
\hline Promedio carga impositiva (T) & 24.449 & 52.701 & 172.249 & 539.432 & 962.668 & 1.869 .311 \\
\hline Desviación estándar de T & 69.716 & 139.844 & 364.902 & 1.237 .121 & 1.410 .928 & 2.539 .813 \\
\hline
\end{tabular}

Fuente: Cálculos propios a partir de la ECP, 2005.

- El parámetro $\sum \theta_{\mathrm{j}}^{\mathrm{OB}}$ se asimila como el valor de la Línea de pobreza, que para el año 2004 correspondía a 1.059 .967 pesos mensuales por hogar ${ }^{11}$.

- $\quad$ El parámetro $\Sigma \theta_{j}^{\mathrm{CV}}$ se asume arbitrariamente (por su subjetividad) utilizando dos valores hipotéticos para interpretación: \$0 y $\$ 300.000$. En la práctica esto es el gasto de subsistencia en arrendamiento (imputado o efectivo) mensual.

- Para aislar las distorsiones de mercado, se calculan los recursos necesarios $(\mathrm{Tr}+\mathrm{OI})$, que garantizan niveles óptimos de consumo de vivienda, bajo el supuesto de que los subsidios y financiamiento son no condicionados.

\subsection{Estimación del modelo y cálculos}

En la sección anterior se definieron las ecuaciones (7 y 8) como las soluciones de política óptima; ahora, para efectos de estimación empírica y con base en los supuestos recientemente descritos, expresamos la ecuación (8) de forma reducida.

$$
T=(\alpha-1)(T r+O I)+\alpha\left(Y-\sum \theta_{j}^{O B}\right)+\beta \sum \theta_{i}^{C V}
$$

11 El dato es tomado de DANE-MERPD. dado que la línea de pobreza incluye gastos de vivienda sería necesario descontar éste para llegar al consumo mínimo de otros bienes. No obstante, para efectos del trabajo decidimos abstraernos de la subjetividad sobre el cálculo de la línea de pobreza y por lo tanto no entrar a debatir en torno a la porción de este ingreso que se dirige al gasto en vivienda. Es así, que el gasto mínimo en vivienda también se asume arbitrariamente, sin ser esto un limitante para interpretar los resultados.
De forma reducida:

$$
T=\varphi+\alpha Y_{n}
$$

Donde

$$
\begin{gathered}
\varphi=(\alpha-1)\left(\operatorname{Tr}+O I-\sum \theta_{i}{ }^{C V}\right) \\
Y n=Y-\sum \theta_{j}{ }^{O B}
\end{gathered}
$$

El objetivo final de este modelo es determinar la necesidad de financiamiento que permita el mayor bienestar de la población, esto es el nivel de transferencias ${ }^{12}$, subsidios $\mathrm{u}$ opciones de financiamiento necesarios para una política óptima $(\mathrm{Tr}+\mathrm{OI})$.

Una estimación por el método de mínimos cuadrados ordinarios (o cualquier alternativa de pronóstico lineal) nos permite obtener una aproximación a los coeficientes $\varphi$ y $\alpha$, los que a su vez nos proporcionan información suficiente para calcular por residuo el financiamiento necesario $(\mathrm{Tr}+\mathrm{O})$. Por despeje algebraico de la expresión (12) es equivalente a la siguiente:

$$
\operatorname{Tr}+O I-=\frac{\varphi}{\alpha-1}+\sum \theta_{i}^{C V}
$$

\subsection{Interpretación}

Los coeficientes estimados $\varphi$ y $\alpha$ representan valores numéricos para los cuales se cumple una relaEs necesario anotar que aquí se trata de transferencias con-
dicionadas por parte del gobierno central. 
ción lineal entre el ingreso neto del gasto mínimo en otros bienes $(Y n)$ y la carga impositiva de un hogar representativo $(T)$. Así, $\varphi$ constituye el nivel teórico de impuestos pagados por el hogar independientemente de su ingreso, mientras que $\alpha$ es el impuesto que paga el hogar por cada peso que su renta excede el gasto mínimo en otros bienes.

Sin embargo, más allá del significado teórico de $\varphi$ y $\alpha$, en el marco de este análisis, su principal utilidad reside en estimar el valor $(\mathrm{Tr}+\mathrm{OI})$, que es el financiamiento (subsidio) requerido para que cada hogar logre consumir niveles óptimos de vivienda de acuerdo a sus preferencias. Podemos considerar los siguientes casos:

- $\quad \mathrm{Si}(\operatorname{Tr}+O I)<0$, no es necesario subsidiar al hogar, por el contrario, dicho hogar estará en la disposición de autofinanciar su consumo de vivienda mínimo y consumir por niveles mayores al mismo.

- $\quad \mathrm{Si}(\mathrm{Tr}+\mathrm{OI})=0$, no es necesario subsidiar al hogar, pero dicho hogar estará en la disposición de autofinanciar justamente su consumo de vivienda mínimo.

- $\quad \mathrm{Si}(\mathrm{Tr}+\mathrm{OI})>0$, es necesario subsidiar al hogar debido a que el consumo de vivienda mínimo supera su disposición a gastar en éste.

Obsérvese que los resultados están en función del ingreso del hogar y del gasto mínimo en vivienda. Este último no deja de ser un valor subjetivo que no ha sido determinado plenamente por los hacedores de política ni la academia, de ahí que sin pérdida de generalidad, en los resultados que siguen se asumen arbitrariamente.

\subsection{Resultados}

Los escenarios a evaluar para estimar los recursos necesarios y la relación entre carga impositiva y nivel de ingresos son los siguientes: i) Hogar bogotano promedio (Total de la población); ii) Hogar representativo estrato 1 ; iii) Hogar representativo estrato 2; iv) Hogar representativo estrato 3; v) Hogar representativo estrato 4; vi) Hogar representativo estrato 5; y vii) Hogar representativo estrato 6.

Tabla 3. Parámetros estimados por tipo de hogar

\begin{tabular}{|c|c|c|c|c|}
\hline Tipo de Hogar & $\alpha$ & $\varphi$ & $\begin{array}{l}T r+01 \text { si } \sum \theta_{\dot{b}}^{c v}=0 \\
\quad \text { (Pesos de 2004) }\end{array}$ & $\begin{array}{c}T r+01 \text { si } \sum \theta_{j}^{c V}=300000 \\
\text { (Pesos de 2004) }\end{array}$ \\
\hline Media Bogotá & $\begin{array}{c}0,18140 * * * \\
(0,01742)\end{array}$ & $\begin{array}{c}103.100^{* * *} \\
(0,00019)\end{array}$ & -125.947 & 174.053 \\
\hline Estrato 1 & $\begin{array}{c}0,01929 * * * \\
(0,00000)\end{array}$ & $\begin{array}{c}29.130 * * * \\
(0,00005)\end{array}$ & -29.703 & 270.297 \\
\hline Estrato 2 & $\begin{array}{c}0,03931^{* * *} \\
(0,00000)\end{array}$ & $\begin{array}{l}54.320 * * * \\
(0,00000)\end{array}$ & -56.543 & 243.457 \\
\hline Estrato 3 & $\begin{array}{c}0,06813^{* * *} \\
(0,00000)\end{array}$ & $\begin{array}{c}125.000 * * * \\
(0,00000)\end{array}$ & -134.139 & 165.861 \\
\hline Estrato 4 & $\begin{array}{c}0,12070^{* * *} \\
(0,00000)\end{array}$ & $\begin{array}{c}241.300^{* * *} \\
(0,00000)\end{array}$ & -274.423 & 25.577 \\
\hline Estrato 5 & $\begin{array}{c}0,12480^{* * *} \\
(0,00004)\end{array}$ & $\begin{array}{c}459.700^{* * *} \\
(0,00000)\end{array}$ & -525.251 & -225.251 \\
\hline Estrato 6 & $\begin{array}{c}0,00511 \\
(0,658)\end{array}$ & $\begin{array}{c}1.674 .000^{\star * *} \\
(0,00000)\end{array}$ & -1.682 .603 & -1.382 .603 \\
\hline
\end{tabular}

*** Indica significancia estadística al 1\%. Los errores estándar incluidos en paréntesis.

Los parámetros fueron estimados por mínimos cuadrados ordinarios.

Fuente: Elaboración propia. 
Los resultados de los coeficientes estimados, así como el cálculo de $(\mathrm{Tr}+\mathrm{OI})$, se resumen en la Tabla 3. Las dos últimas columnas presentan los recursos adicionales necesarios para financiar el consumo de vivienda óptimo de cada hogar bajo dos conjeturas: la primera, si el consumo de vivienda mínimo o de subsistencia es cero, lo cual sólo es un referente pues evidentemente es positivo; la segunda, asume arbitrariamente que el consumo de subsistencia es de $\$ 300.000$ a precios de $2004^{13}$. Los recursos adicionales necesarios en esta última conjetura son positivos para los hogares de estrato 1 hasta el 4 , esto es, implica subsidiar y/o financiar a los hogares de estrato 4 inclusive.

La Tabla 4 ilustra los resultados de la penúltima columna de la Tabla 3 pero a precios de 2015 tras realizar la deflactación de dichos valores. A continuación se destaca el resultado básico de cada uno de los escenarios o tipos de hogar.

Escenario i). Un individuo representativo de la población bogotana destinará recursos para sus condiciones de habitabilidad por $\$ 282.705$ mensuales (precios de 2015), su diferencia con $\Sigma \theta_{i}^{\mathrm{CV}}$ deberá ser el monto de demanda a subsidiar por el estado o por otra fuente de financiación.

Escenario ii). Un individuo representativo de estrato 1 destinará recursos para sus condiciones de habitabilidad por $\$ 66.673$ mensuales. Cualquier consumo de vivienda mayor requiere apoyo en financiación.

Escenario iii). Un individuo representativo de estrato 2 destinará recursos para sus condiciones de habitabilidad por $\$ 126.919$ mensuales. Cualquier consumo de vivienda mayor requiere apoyo en financiación.

Escenario iv). Un individuo representativo de estrato 3 destinará recursos para sus condiciones de habitabilidad por $\$ 301.093$ mensuales. Cualquier

13 El nivel de consumo de subsistencia es un valor difícil de establecer en el contexto de este trabajo. Es necesario utilizar criterios técnicos y sociales para medir dicho valor, lo cual rebasa el objetivo de este documento. No obstante, el valor de $\$ 300.000$ equivale cercanamente al 30\% de la línea de pobreza, de ahí que puede ser un buen referente de análisis. consumo de vivienda mayor requiere apoyo en financiación.

Escenario v). Un individuo representativo de estrato 4 destinará recursos para sus condiciones de habitabilidad por $\$ 615.979$ mensuales. Cualquier consumo de vivienda mayor requiere apoyo en financiación.

Escenario vi). Un individuo representativo de estrato 5 destinará recursos para sus condiciones de habitabilidad por \$1.178.997 mensuales. Cualquier consumo de vivienda mayor requiere apoyo en financiación.

Escenario vii). Un individuo representativo de estrato 6 destinará recursos para sus condiciones de habitabilidad por $\$ 3.776 .829$ mensuales. Cualquier consumo de vivienda mayor requiere apoyo en financiación.

Tabla 4. Consumo de vivienda según tipo de hogar

\begin{tabular}{|c|c|}
\hline Tipo de Hogar & $\begin{array}{c}\text { Disposición mensual a pagar } \\
\text { (Pesos de 2015) }\end{array}$ \\
\hline Media Bogotá & $\$ 282.705$ \\
\hline Estrato 1 & $\$ 66.673$ \\
\hline Estrato 2 & $\$ 126.919$ \\
\hline Estrato 3 & $\$ 301.093$ \\
\hline Estrato 4 & $\$ 615.979$ \\
\hline Estrato 5 & $\$ 1.178 .997$ \\
\hline Estrato 6 & $\$ 3.776 .829$ \\
\hline
\end{tabular}

Fuente: Cálculo de los autores.

La llustración 4 muestra los resultados de las estimaciones y refleja la brecha de cada escenario con el consumo de vivienda de subsistencia (hipotéticamente de $\$ 300.000$ a precios de 2004). La brecha para los hogares que se encuentren con un consumo de vivienda inferior a $\theta_{i}{ }^{C V}$ denota la cuantía de recursos necesarios para garantizar dichos niveles mínimos.

Se observa así que un hogar estrato 1 presenta una disposición a pagar de tan sólo $\$ 29.700$ a precios de 2004, muy inferior a un hipotético valor de $\$ 300.000$. Más allá de la discusión sobre el verdadero valor que le permitiría a dicho hogar satisfacer sus necesidades básicas de vivienda, se evidencia la necesidad de una política social de vivienda que 
permita que estos hogares con tan baja disposición a pagar logren acceder a estos niveles mínimos ${ }^{14}$.

Los resultados de este ejercicio son explicativos de la gran problemática social que ha enfrentado la ciudad en torno a la calidad de vivienda de su población, sugiriendo que tan sólo los estratos 5 y 6 no requieren apoyo gubernamental para lograr una calidad de vivienda adecuada. Esto se suma a que, según datos del DANE y la Secretaría Distrital de Planeación (SDP), sólo el 5,6\% de los hogares Bogotanos pertenecen a esos estratos sociales (125.149 hogares de un total de 2.251.170). Así, si tomamos estos resultados como certeros, entonces 2.126.021 hogares de la ciudad requieren recursos adicionales para asegurar niveles óptimos de gasto en vivienda, de ahí que políticas como la entrega de viviendas gratis, por ejemplo, resultarán claramente insuficientes.

Otro resultado significativo es la amplia brecha existente entre los diferentes estratos de la población Bogotana (llustración 4). La discusión admite diferentes dimensiones, $e$ incluso algunas voces sugieren la posibilidad, dado el aparente surgimiento de sectores con una disposición excesivamente alta a pagar por vivienda, de incorporar un nuevo estrato a la ciudad (estrato 7). Lo cierto, es que esta dinámica es evidencia de la alta segregación social y de los numerosos matices que debe tener en cuenta cualquier política de vivienda.

\section{Conclusiones y recomendaciones}

La política de vivienda en Colombia se ha centrado en mecanismos de financiación subsidiada, desconociendo que dicho consumo va más allá de la compra de vivienda nueva o usada. Es por eso que en este trabajo se ha definido el consumo de vivienda como aquél gasto en el que incurre un hogar periódicamente (mensualmente por lo general) para solucionar su necesidad de techo. La compra de vivienda es solo una alternativa de consumo de vivienda, pues el arrendamiento efectivo

14 Es importante destacar que ante la insuficiente disponibilidad de información actualizada y la subjetividad de la medición en el valor de la línea de pobreza, gasto de subsistencia, etc., los valores estimados solo reflejan aproximaciones imperfectas de una medición real. Asimismo, más que el ejercicio econométrico utilizado, se quiere destacar el resultado acerca de la dificultad financiera de sostener una política limitada a la demanda. es otra posibilidad para suplir esta necesidad, sin embargo, la compra se ha constituido en un mecanismo para generar propiedad y rentas a las clases populares.

La política de vivienda en los últimos años, así como la evolución de los indicadores del mercado demuestran que el enfoque de dicha política es de estímulo a la demanda, relegando la búsqueda de un crecimiento urbanístico sostenible para la ciudad. Los gobiernos optan por este tipo de medidas debido a que el alcance de las políticas de oferta es de largo plazo y no son visibles en el gobierno de turno. No obstante, en términos macroeconómicos una política de subsidios es insostenible e ineficiente por las características socioeconómicas de la población bogotana. Una asignación óptima, teóricamente, podría implicar subsidios incluso para población de estrato 4 , por lo tanto, los niveles de financiación necesarios para una política de demanda pueden resultar excesivamente altos, con lo que se justifica la pertinencia de una política orientada al mejoramiento y el aprovechamiento eficiente de la oferta actual, más que seguir concentrando esfuerzos en fomentar la demanda.

Finalmente, el análisis formal del mercado sugiere que la política efectuada hasta ahora es insuficiente para garantizar un consumo de vivienda mínimo a toda la población, e incluso tiende a distorsionar el consumo de los hogares. La vivienda se encuentra sobrevalorada y los demás bienes subvalorados en términos de consumo, vía efecto sustitución, por cuenta de la política subsidiaria, favoreciendo así la rentabilidad de los constructores y propietarios, más no necesariamente los hogares candidatos a comprar vivienda.

El modelo propuesto permitió obtener algunos valores que dan luces sobre las características de la población bogotana y las brechas existentes entre estratos socioeconómicos. Se observa por ejemplo que la disposición a efectuar gasto en vivienda de los hogares de estrato 6 es cerca de 57 veces la disposición de los hogares de estrato 1 . Sin embargo, al ser estático nuestro modelo y limitado en el uso de variables, es conveniente una segunda fase de investigación que haga uso de un modelo en forma ampliada incorporando dotaciones iniciales en términos de vivienda, así como capacidades productivas y financieras de la población. 
Ilustración 4. Demanda de vivienda óptima según estratos socioeconómicos

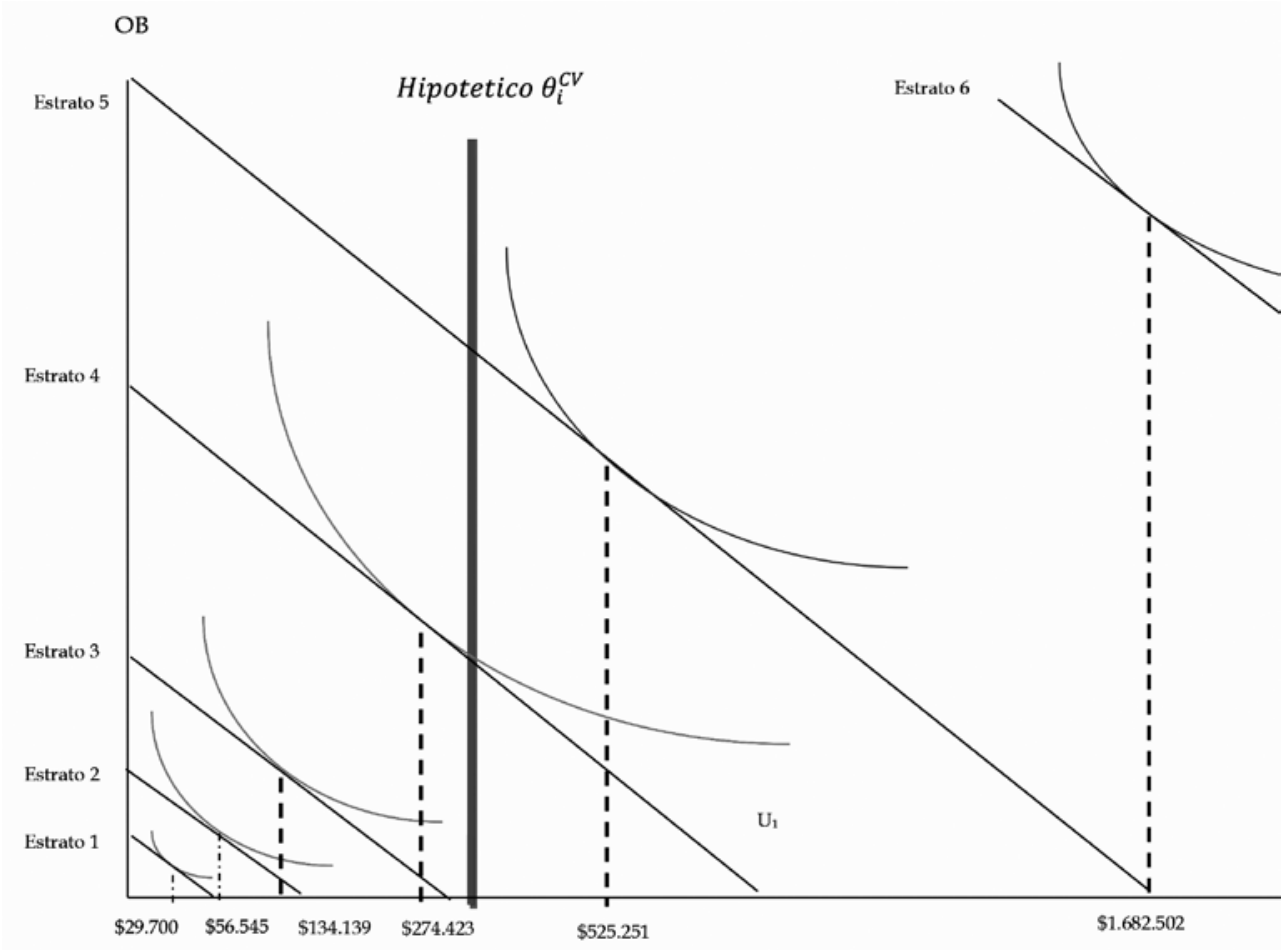

Fuente: Elaboración propia.

\section{Referencias}

Achury, C. Hubar, S. \& Koulovatianos, C. (2012). Saving rates and portfolio choice with subsistence consumption. Review of Economic Dynamics, 15(1), 108-126.

Ando, A. \& Modigliani, F. (1963). The "Life-Cycle" Hypothesis of Savings: Aggregate implications and tests. American Economic Review, 53, 55-84.

Awan, K., Odling-Smee, J. \& Whitehead, C. (1982). Household attributes and the demand for private rental housing. Economica, 49, 183-200.

Biorn, E. (1980). The Consumption Function and the Life-Cycle Hypothesis: An Analysis of Norwegian Household Data. The Scandinavian Journal of Economics, 82(4), 464-480.

Black, D. (1958). The theory of Committees and Elections, Cambridge.

Camelo, M. (2009). Sobre el desarrollo de la Teoría de los Bienes Públicos Locales. Revista Finanzas y Política Económica, 1 (2), 35-52.

Camelo, M. \& Campo, J. (2016). Análisis de la política de vivienda en Bogotá: un enfoque desde la oferta y la demanda. Revista Finanzas y Política Económica, 8, 105-122.
Cocco, J. (2005). Portfolio Choice in the Presence of Housing. Review of Financial Studies, 18, 535-567.

De Borger, L. (1984). Household Attributes and the Demand for Private Rental Housing: Comment. Economica, New Series, 51 (203), 351-355.

De Borger, L. (1986). Estimating the benefits of public-housing prorams: a characteristics approach. Journal of regional science, 20(4).

Downs, A. (1957). Economic Theory of Political Action in a Democracy. Journal of Political Economy, 65.

Echeverri, J., Garcia, O. \& Pineda, B. (1999). UPAC: evolución y crisis de un modelo de desarrollo. Bogotá: Departamento Nacional de Planeación, Archivos de Macroeconomía, 128.

Epple, D. \& Visscher, M. (1978). A Search for Testable Implications of the Tiebout Hypothesis. Politica Economica, 86, 405-25.

Gómez, J., Ojeda, J., Rey, C. \& Sicard, N. (2015). Detectando burbujas en el mercado de vivienda Colombiano: Un nuevo enfoque. Desarrollo y Sociedad, 75 (24), 197-222.

Hall, R. (1978). Stochastic Implications of the Life Cycle-Permanent Income Hypothesis: Theory and evidence. Journal of Political Economy, 86, 971- 987. 
Hamilton, B. (1975). Zoning and Property Taxation in a System of Local Governments. Urban Studies, 12, 205- 11.

Henderson, J. (1979). Theories of group, jurisdiction, and city size. Current issues in urban economics. In: P. Mieskowski \& M. Straszheim (eds). The Johns Hopkins University Press, Baltimore, 235-269.

Jaramillo, S. (1992). La vivienda en Bogotá. Evolución de las condiciones habitacionales y de la estructura de producción de vivienda. Documento CEDE, 87. Bogotá.

López, E. \& Salamanca, A. (2009). El efecto riqueza de la vivienda en Colombia”. Borradores de Economía, 551. Banco de la Republica de Colombia.

Primera Encuesta Multipropósito para Bogotá - EMB (2011). Secretaría de Planeación Distrital. Alcaldía Mayor de Bogotá.

Renzetti, S., Dupont, D. \& Chitsinde, T. (2015). An empirical examination of the distributional impacts of water pricing reforms. Utilities Policy, 34.

Rubinfeld, D. (1987). The economics of the local public sector. Handbook of Public Economics, 2, 571-645.

Samuelson, P. (1954). The Pure Theory of Public Expenditure. The Review of Economics and Statistics, 36(4), 387-389.

Sánchez, V. \& Vega, C. (2015). ¿Hay una burbuja inmobiliaria en Bogotá? Un estudio por segmentos de Mercado. Revista de Economia Institucional, 17 (32), 233-257
Schmidheiny, K. (2006). Income segregation from local income taxation when households differ in both preferences and incomes. Regional Science and Urban Economics, 36, 270-299.

Smith, L., Rosen, K. \& Fallis, G. (1988). Recent Developments in Economic Models of Housing Markets. Journal of Economic Literature, 26 (1), 29-64.

Stone, R. (1954). Linear Expediture System and Demand Analysis: An application to the Pattern of British Demand. Economic Journal, 64.

Stone, R. (2014). Models for demand projections. Essays on Econometrics and Planning, 271-290.

Tiebout, C. (1956). A Pure Theory of Local Expediture. The Journal of Political Economy, 64 (5), 416-424.

Urrutia, M. \& Namen, O. (2012). Historia del crédito hipotecario en Colombia. Ensayos sobre política económica, 30(67).

Vergara, R. (2008). El mejoramiento de vivienda y entorno en Bogotá, 1994-1998: programas, conceptos y estrategias. Revista Investigación y Desarrollo, 16(1), 196-225.

Yinger, J. (1980). Capitalization and the Theory of Local Public Finance. Disc. Paper D80-7, Kennedy School of Government, Harvard Univ.

Yinger, J. et al. (1988). Property Taxes and House Values: The Theory and Estimation of Intrajurisdictional Property Tax Capitalization. Boston: Academic Press. 


\section{ANEXO}

Tabla 1. Estimación parámetros según tipo de hogar

\begin{tabular}{|c|c|c|c|c|}
\hline \multicolumn{5}{|c|}{ Hogar Bogotano representativo } \\
\hline & Coeficiente & Error estándar & Estadístico t & P-Valor \\
\hline Constante & 103100 & 19320 & 5.334 & 0.0001 \\
\hline Yn & 0.1814 & 0.02 & 10.412 & 0.0000 \\
\hline $\mathrm{R}$ cuadrado & 0.8645 & & & \\
\hline R cuadrado ajustado & 0.8565 & & & \\
\hline Estadístico $\mathrm{F}(1,17)$ & 108.4 & & & 0.0000 \\
\hline \multicolumn{5}{|c|}{ Hogar Estrato 1} \\
\hline & Coeficiente & Error estándar & Estadístico $\mathrm{t}$ & P-Valor \\
\hline Constante & 29130 & 1275 & 22.84 & 0.0000 \\
\hline$Y n$ & 0.0193 & 0.0021 & 9.384 & 0.0000 \\
\hline $\mathrm{R}$ cuadrado & 0.03719 & & & \\
\hline $\mathrm{R}$ cuadrado ajustado & 0.03677 & & & \\
\hline Estadístico $\mathrm{F}(1,2280)$ & 88,07 & & & 0.0000 \\
\hline \multicolumn{5}{|c|}{ Hogar Estrato 2} \\
\hline & Coeficiente & Error estándar & Estadístico t & P-Valor \\
\hline Constante & 54320 & 1400 & 38.79 & 0.0000 \\
\hline $\mathrm{Yn}$ & 0.03931 & 0.0019 & 21.15 & 0.0000 \\
\hline $\mathrm{R}$ cuadrado & 0.08253 & & & \\
\hline $\mathrm{R}$ cuadrado ajustado & 0.08235 & & & \\
\hline Estadístico F(1,4973) & 447,3 & & & 0.0000 \\
\hline \multicolumn{5}{|c|}{ Hogar Estrato 3} \\
\hline & Coeficiente & Error estándar & Estadístico $\mathrm{t}$ & P-Valor \\
\hline Constante & 125000 & 4464 & 28.01 & 0.0000 \\
\hline Yn & 0.06813 & 0.0029 & 23.72 & 0.0000 \\
\hline $\mathrm{R}$ cuadrado & 0.1366 & & & \\
\hline R cuadrado ajustado & 0.1363 & & & \\
\hline Estadístico F(1,3559) & 562,9 & & & 0.0000 \\
\hline \multicolumn{5}{|c|}{ Hogar Estrato 4} \\
\hline & Coeficiente & Error estándar & Estadístico t & P-Valor \\
\hline Constante & 241300 & 54040 & 4.464 & 0.0000 \\
\hline$Y n$ & 0.1207 & 0.0134 & 9.02 & 0.0000 \\
\hline $\mathrm{R}$ cuadrado & 0.09727 & & & \\
\hline $\mathrm{R}$ cuadrado ajustado & 0.09608 & & & \\
\hline Estadístico F(1,755) & 81,35 & & & 0.0000 \\
\hline
\end{tabular}


VALORACIÓN DE LOS SERVICIOS PÚBLICOS DOMICILIARIOS DE CARTAGENA DE INDIAS (COLOMBIA)

\begin{tabular}{|c|c|c|c|c|}
\hline \multicolumn{5}{|c|}{ Hogar Estrato 5} \\
\hline & Coeficiente & Error estándar & Estadístico t & P-Valor \\
\hline Constante & 459700 & 106600 & 4.312 & 0.0000 \\
\hline$Y n$ & 0.1248 & 0.0197 & 6.346 & 0.0000 \\
\hline R cuadrado & 0.1032 & & & \\
\hline $\mathrm{R}$ cuadrado ajustado & 0.1006 & & & \\
\hline Estadístico F(1,350) & 40,27 & & & 0.0000 \\
\hline \multicolumn{5}{|c|}{ Hogar Estrato 6} \\
\hline & Coeficiente & Error estándar & Estadístico t & P-Valor \\
\hline Constante & 1674000 & 125200 & 13.372 & 0.0000 \\
\hline$Y n$ & 0.0005113 & 0.0012 & 0.443 & 0.6580 \\
\hline R cuadrado & 0.001017 & & & \\
\hline $\mathrm{R}$ cuadrado ajustado & 0.004159 & & & \\
\hline Estadístico F(1,193) & 0,1964 & & & 0,6581 \\
\hline
\end{tabular}

